\title{
ON SOME CRITERIA OF CARLEMAN FOR THE COMPLETE CONVERGENCE OF A $J$-FRACTION
}

\author{
H. S. WALL
}

1. Introduction. Carleman $[1, \mathrm{pp} .214-215]^{1}$ derived, from his theory of integral equations, a number of criteria for the complete convergence of a real $J$-fraction

$$
-\varlimsup_{p=1}^{\infty} \frac{-a_{p-1}^{2}}{b_{p}+z} \quad\left(a_{0}=1, a_{p} \neq 0\right) .
$$

The most important one of these criteria states that the $J$-fraction is completely convergent if the series $\sum\left(1 / c_{2 p}\right)^{1 / 2 p}$ diverges, where $c_{0}, c_{1}, c_{2}, \cdots$ are the coefficients in the power series $\sum\left(c_{p} / z^{p+1}\right)$ associated with the $J$-fraction. In [2] Carleman gave an algebraic proof of this theorem for the case where $b_{p}=0, p=1,2,3, \cdots$. The present note contains an algebraic proof for the general case, and some remarks concerning the other criteria of Carleman, especially with reference to their application to $J$-fractions with arbitrary complex coefficients.

2. The determinate and indeterminate cases. Let $a_{0}=1, a_{p} \neq 0$, $b_{p}, p=1,2,3, \cdots$, be complex constants, and consider the system of linear equations

$$
-a_{p-1} x_{p-1}+\left(b_{p}+z\right) x_{p}-a_{p} x_{p+1}=0, \quad p=1,2,3, \cdots .
$$

Since the $a_{p}$ are not zero, these equations determine $x_{2}, x_{3}, x_{4}, \ldots$ uniquely in terms of arbitrarily chosen initial values $x_{0}, x_{1}$. If $x_{0}=-1$, $x_{1}=0$, let $x_{p}=X_{p}(z)$, and if $x_{0}=0, x_{1}=1$, let $x_{p}=Y_{p}(z)$. Then, $X_{p+1}(z) / Y_{p+1}(z)$ is the $p$ th approximant of the $J$-fraction (1.1). If the infinite series $\sum\left|X_{p}(z)\right|^{2}$ and $\sum\left|Y_{p}(z)\right|^{2}$ both converge for one value of $z$, then they converge for every value of $z[7, \mathrm{p} .120]$. We may accordingly distinguish two cases for a $J$-fraction (1.1) with complex coefficients. In the indeterminate case, both the infinite series

$$
\sum\left|X_{p}(0)\right|^{2}, \quad \sum\left|Y_{p}(0)\right|^{2}
$$

are convergent. In the determinate case, at least one of these infinite series is divergent. A real $J$-fraction is completely convergent if and only if the determinate case holds. This is also the case of a determinate moment problem [4].

Received by the editors July $31,1947$.

1 Numbers in brackets refer to the bibliography at the end of the paper. 
On applying Schwarz's inequality to the determinant formulas

$$
X_{p+1}(z) Y_{p}(z)-X_{p}(z) Y_{p+1}(z)=\frac{1}{a_{p}}
$$

and

$$
X_{p+2}(z) Y_{p}(z)-X_{p}(z) Y_{p+2}(z)=\frac{b_{p+1}+z}{a_{p} a_{p+1}},
$$

after setting $z=0$, we find immediately that when the indeterminate case holds then the series $\sum\left(1 /\left|a_{p}\right|\right)$ and $\sum\left|b_{p+1} / a_{p} a_{p+1}\right|$ are convergent. These and other criteria obtained by applying Schwarz's inequality to other determinant formulas were found by Dennis and Wall [3]. They were not aware of the fact that Carleman [1, p. 215] had given the first of these criteria for the case of real $J$-fractions. Hellinger [6] showed that the determinate case holds for a real $J$-fraction if lim inf $\left|a_{p}\right|$ is finite. This criterion, which is contained in the first criterion mentioned above, was given by Carleman $[1, p$. 215].

Let $\delta_{1}, \delta_{2}, \delta_{3}, \cdots$ be complex numbers such that $\delta \leqq\left|\delta_{p}\right| \leqq \Delta$, $p=1,2,3, \cdots$, where $\delta$ and $\Delta$ are positive constants independent of $p$. If the determinate case holds for the J-fraction (1.1), then the determinate case holds for the J-fraction

$$
-\varlimsup_{p=1}^{\infty} \frac{-a_{p-1}^{2} \delta_{p-1} \delta_{p}}{b_{p} \delta_{p}+z} \quad\left(\delta_{0}=1\right) .
$$

The introduction of the factors $\delta_{k}$ into (1.1) effects the replacement of the series (2.2) by the series

$$
\sum\left|\frac{\delta_{1}}{\delta_{p}}\right|\left|X_{p}(0)\right|^{2}, \quad \sum\left|\frac{\delta_{1}}{\delta_{p+1}}\right|\left|Y_{p}(0)\right|^{2}
$$

so that the theorem is obviously true. Carleman $[1$, p. 215] obtained this for the case of real $a_{p}, b_{p}, \delta_{p}$ as a corollary to his general theory.

Let $\alpha_{p}$ and $\beta_{p}$ be complex numbers such that

$$
\left|\alpha_{p}\right|<M, \quad\left|\beta_{p}\right|<M, \quad a_{p}+\alpha_{p} \neq 0, p=1,2,3, \cdots .
$$

If the determinate case holds for the $J$-fraction (1.1), then the determinate case holds for the $J$-fraction

$$
-K_{p=1}^{\infty} \frac{-\left(a_{p-1}+\alpha_{p-1}\right)^{2}}{b_{p}+\beta_{p}+z} \quad\left(\alpha_{0}=0\right) .
$$

Carleman $\left[1\right.$, p. 214] gave this theorem for the case of real $a_{k}, b_{k}$, 
$\alpha_{k}, \beta_{k}$. If these numbers are complex and $\alpha_{k}=0, k=1,2,3, \cdots$, then the theorem can be proved by means of an easy modification of the proof given in [7, pp. 120-121]. (Cf. also [8, p. 557].) The method used in [7] can be modified to cover the case $\beta_{k}=0, k=1,2,3, \ldots$, and hence to establish the theorem in the general case. We shall indicate briefly those modifications. Let

$$
L_{p}(x)=-a_{p-1} x_{p-1}+b_{p} x_{p}-a_{p} x_{p+1}, \quad p=1,2,3, \cdots,
$$

and let $L_{p}^{\prime}(x)$ denote the expression $L_{p}(x)$ in which $a_{p}$ is replaced by $a_{p}+\alpha_{p}$, where the $\alpha_{p}$ are any complex constants such that $\left|\alpha_{p}\right|<M$, $a_{p}+\alpha_{p} \neq 0, p=1,2,3, \cdots$. The solution of the system $L_{p}(x)=0$ under the initial conditions $x_{0}=-1, x_{1}=0$ is $x_{p}=X_{p}(0)$, and under the initial conditions $x_{0}=0, x_{1}=1$ the solution is $x_{p}=Y_{p}(0)$. Let $x_{p}^{\prime}$ be the solution of the system $L_{p}^{\prime}(x)=0$ under the initial conditions $x_{0}^{\prime}=a, x_{1}^{\prime}=b$, where $a$ and $b$ are arbitrarily chosen constants. The theorem will be established if we show that the convergence of the series $\sum\left|X_{p}(0)\right|^{2}$ and $\sum\left|Y_{p}(0)\right|^{2}$ implies the convergence of the series $\sum\left|x_{p}^{\prime}\right|^{2}$.

If $\xi_{p}$ and $\xi_{p}^{\prime}$ are arbitrary solutions of the systems $L_{p}=0$ and $L_{p}^{\prime}=0$, respectively, then we have the Green's formula

$$
\begin{aligned}
\sum_{p=1}^{n}\left[\xi_{p} L_{p}^{\prime}\left(\xi^{\prime}\right)-\xi_{p}^{\prime} L_{p}(\xi)\right]-\left[\left(\xi_{p} \xi_{p+1}^{\prime}-\xi_{p}^{\prime} \xi_{p+1}\right) a_{p}\right]_{0}^{n} & \\
& -\sum_{p=1}^{n}\left(\alpha_{p-1} \xi_{p-1}^{\prime}+\alpha_{p} \xi_{p+1}^{\prime}\right) \xi_{p}=0 .
\end{aligned}
$$

If, in particular, $\xi_{p}=X_{p}(0), \xi_{p}^{\prime}=x_{p}^{\prime}$, this gives

$$
-b+\left(x_{n}^{\prime} X_{n+1}-x_{n+1}^{\prime} X_{n}\right) a_{n}-\sum_{p=1}^{n}\left(\alpha_{p-1} x_{p-1}^{\prime}+\alpha_{p} x_{p+1}^{\prime}\right) X_{p}=0,
$$

where we have written $X_{k}$ for $X_{k}(0)$; and if $\xi_{p}=Y_{p}(0)=Y_{p}, \xi_{p}^{\prime}=x_{p}^{\prime}$, we obtain

$$
-a+\left(x_{n}^{\prime} Y_{n+1}-x_{n+1}^{\prime} Y_{n}\right) a_{n}-\sum_{p=1}^{n}\left(\alpha_{p-1} x_{p-1}^{\prime}+\alpha_{p} x_{p+1}^{\prime}\right) Y_{p}=0 .
$$

On multiplying the first of these equations by $Y_{n}$, the second by $-X_{n}$, and adding, we find that

$$
x_{n}^{\prime}-\sum_{p=1}^{n} k_{n, p} x_{p}^{\prime}=h_{n}
$$

where 


$$
\begin{aligned}
k_{n, p}= & \left\{\begin{array}{l}
\frac{\alpha_{p}\left(X_{p+1} Y_{n}-X_{n} Y_{p+1}\right)+\alpha_{p-1}\left(X_{p-1} Y_{n}-X_{n} Y_{p-1}\right)}{1+\left(\alpha_{n-1} / a_{n-1}\right)}, p=1,2, \cdots, n-1, \\
0, \quad p=n,
\end{array}\right. \\
h_{n} & =\frac{b Y_{n}-a X_{n}}{1+\left(\alpha_{n-1} / a_{n-1}\right)} .
\end{aligned}
$$

Under the hypothesis that the series $\sum\left|X_{p}\right|^{2}$ and $\sum\left|Y_{p}\right|^{2}$ converge, it follows, as indicated before, that the series $\sum\left|1 / a_{p}\right|$ converges, so that $\lim _{n-\infty}\left|a_{n-1}\right|=\infty$. Since, by hypothesis, $\left|\alpha_{p}\right|<M$, $p=1,2,3, \cdots$, we then find at once by Schwarz's inequality that the double series $\sum\left|k_{p q}\right|^{2}$ and the series $\sum\left|h_{p}\right|^{2}$ are convergent. It then follows exactly as in [7, p. 121] that the series $\sum\left|x_{p}^{\prime}\right|^{2}$ is convergent, and the theorem is thereby established.

3. Criterion involving the moments $c_{p}$. Let $\sum\left(c_{p} / z^{p+1}\right)$ be the power series expansion in descending powers of $z$ of a real $J$-fraction (1.1). If the series

$$
\sum_{p=1}^{\infty}\left(\frac{1}{c_{2 p}}\right)^{1 / 2 p}
$$

diverges, then the determinate case holds for the $J$-fraction. We shall give a simple algebraic proof of this well known theorem of Carleman $[1$, p. 215].

The quadratic forms

$$
F_{n}=\sum_{p, q=1}^{n} c_{p+q-2} x_{p} x_{q}, \quad n=1,2,3, \cdots,
$$

are all positive definite [4], and we may therefore write

$$
\begin{aligned}
F_{n} \equiv & \left(b_{11} x_{1}+b_{12} x_{2}+\cdots+b_{1 n} x_{n}\right)^{2}+\left(b_{22} x_{2}+b_{23} x_{8}+\cdots+b_{2 n} x_{n}\right)^{2} \\
& +\cdots+\left(b_{n n} x_{n}\right)^{2},
\end{aligned}
$$

where the $b_{p q}$ are real constants independent of $n$ for $p \leqq n, q \leqq n$, and where $b_{p p} \neq 0, p=1,2,3, \cdots, n$. The $b_{p p}$ are connected with the partial numerators of the $J$-fraction by the formulas [9]

$$
\left(a_{0} a_{1} \cdots a_{p}\right)^{2}=b_{p+1, p+1}^{2} \quad\left(a_{0}=b_{11}=1\right)
$$

and, obviously,

$$
c_{2 p}=\sum_{q=1}^{p+1} b_{q, p+1}^{2} \quad(p=0,1,2, \cdots)
$$


so that $c_{2 p} \geqq b_{p+1, p+1}^{2}$. Consequently,

$$
\left(a_{0} a_{1} \cdots a_{p}\right)^{2} \leqq c_{2 p} \text {. }
$$

Hence, by Carleman's inequality ${ }^{2}$ [2],

$$
e \sum_{p=1}^{n} \frac{1}{\left|a_{p}\right|}>\sum_{p=1}^{n}\left(\frac{1}{a_{1}^{2} a_{2}^{2} \cdots a_{p}^{2}}\right)^{1 / 2 p} \geqq \sum_{p=1}^{n}\left(\frac{1}{c_{2 p}}\right)^{1 / 2 p} .
$$

Therefore, if the series (3.1) is divergent, then the series $\sum\left|1 / a_{p}\right|$ is divergent, so that, as indicated in $\$ 2$, the determinate case holds for the $J$-fraction.

\section{BIBLIOGRAPHY}

1. T. Carleman, Sur les équations integrales singulieres a noyau rél et symetrique, Uppsala, 1923.

2. - Les fonctions quasi analytiques, Paris, 1926.

3. J. J. Dennis and H. S. Wall, The limit-circle case for a positive definite J-fraction, Duke Math. J. vol. 12 (1945) pp. 255-273.

4. H. Hamburger, Über eine Erweiterung des Stieltjesschen Momentenproblems, Math. Ann. vol 81 (1920) pp 235-319; vol. 82 (1920) pp. 120-164 and 168-187.

5. G. H. Hardy, J. E. Littlewood and G. Polya, Inequalities, Cambridge, 1934.

6. E. Hellinger, Zur Stieltjesschen Kettenbruchtheorie, Math. Ann. vol. 86 (1922) pp. 18-29.

7. E. Hellinger and H. S. Wall, Contributions to the analytic theory of continued fractions and infinite matrices, Ann. of Math. vol. 44 (1943) pp. 103-127.

8. W. T. Scott and H. S. Wall, On the convergence and divergence of continued fractions, Amer. J. Math. vol. 69 (1947) pp. 551-561.

9. T. J. Stieltjes, Sur la réduction en fraction continue d'une série procédant suivant es puissances descendantes d'une variable, Oeuvres, vol. 2, pp. 184-200.

The University of Texas

${ }^{2}$ For an algebraic proof of Carleman's inequality see, for instance [5]. 